\title{
Prader-Willi syndrome: genotype, cause, phenotype and management
}

\section{Abstract}

Prader-Willi syndrome (PWS) is a complex neurodevelopmental genetic condition that results in a range of phenotypic features including hypotonia, hyperphagia and behavioural difficulties. PWS is caused by the paternal loss of imprinting genes from the chromosome 15q11.2-13. If left unmanaged, the central obesity caused by hyperphagia and the behavioural features such as foraging and stealing of foods will dominate the life of the person with PWS and his/her family, resulting in practical and psychological difficulties. In this article, Delia Pogson outlines the pathogenesis of this rare disorder and discusses the multidisciplinary approach required to manage and treat this condition.

Delia Pogson is Senior Lecturer, Faculty of Health Sciences, University of Southampton

\section{Key words \\ - Prader-Willi syndrome \\ - Genomic imprinting \\ - Hyperphagia \\ - Central obesity \\ - Intellectual disability \\ This article has been subject to double-blind peer review}

$\mathrm{P}$ rader-Willi syndrome (PWS) is a complex neurodevelopmental genetic condition that was first described in 1956 by Prader and colleagues. The condition is characterized by a recognizable pattern of dysmorphic features as well as major neurological, developmental, behavioural and psychiatric differences (Cassidy and Driscoll, 2009; Butler, 2011; McAllister et al, 2011; McCandless and The Committee on Genetics, 2011; Prader-Willi Syndrome Association UK (PWSA(UK)), 2012). It has been highlighted that PWS should be referred to as a genomic condition rather than a genetic condition because of its genomic imprinting feature (Cassidy and Driscoll, 2009; McCandless and The Committee on Genetics, 2011).

\section{The genotype of PWS}

Each cell in the human body contains genetic material inside its nucleus in the form of chromosomes (the genotype); 23 pairs of chromosomes composed of two strands of deoxyribonucleic acid (DNA) are linked together to form a 'double helix', with one chromosome from each pair being inherited from each parent. The DNA genes (segments of the DNA) provide the genetic code for specific amino acids, which in turn form specific proteins - the building blocks of the human body. Any disruption or changes to DNA may result in alterations to protein expression and function (the phenotype).
Normally the two copies of genes (one from each parent) are equally controlled, but for a small proportion of genes certain sections of the genome are only activated if they are inherited from a parent of a particular sex. Therefore, for some areas of the genetic material the maternally and paternally derived copies are controlled differently. The term 'imprinting' is used to describe the epigenetic mechanism causing this unequal control of paternal and maternal genes. A small number of genes are subject to genomic imprinting, where a gene from a parent of a particular sex is 'switched off' - silenced or not active as a result of being imprinted.

Imprinted genes tend to be organized in clusters regulated by one or more imprinting centres (ICS). In the case of PWS, the genes on chromosome $15 q 11.2-13$ are subject to this epigenetic modification, and it is only the copy/gene inherited from the father that is normally expressed; the maternal copy is quite normally 'imprinted' and not active. Under normal circumstances, just having this one active copy from the father is sufficient for normal function; however, PWS develops when a person has a deletion or mutation at 15q11.2-13 on the paternal copy and the only active copy of the relevant genes are deleted or lost (Goldstone, 2004; Cassidy and Driscoll, 2009; Butler, 2011; McAllister et al, 2011; McCandless and The Committee on Genetics, 2011; PWSA(UK), 2012) (Figure 1). 
Dykens et al (2011) highlight that the conditions PWS and Angelman syndrome (AS), a condition caused by disruption in the maternally expressed ubiquitin-protein ligase E3A (UBE3A) gene in the same q11-13 region of chromosome 15 , made molecular genetic history as the first disorders to show these effects of parent-oforigin genomic imprinting.

\section{Cause of PWS}

PWS is a complex genomic imprinted condition resulting from the absence of normally active paternally expressed genes from the chromosome 15q11.2-13 region, with a number of underlying causative factors. Deletions in the paternally inherited chromosome 15q11.2-13 region account for $70-75 \%$ of cases; $25-30 \%$ from maternal uniparental disomy (mUPD) and the remaining from mutations or defects of the IC (Cassidy and Driscoll, 2009; Butler, 2011; DuBose et al, 2011; McAllister et al, 2011; McCandless and The Committee on Genetics, 2011).

There are three main subtypes of PWS (Table 1), with the common feature being loss of expression of the paternally-expressed imprinting genes in the critical region.

\section{Deletions}

Deletions are classified according to size: larger type I deletions (BP1-BP3); and smaller type II deletions (BP2-BP3). There are two major proximal chromosome breakpoints (BP1 and BP2) and a distal breakpoint (BP3) in the 15q1113 region (Figure 2 ).

\section{mUPD}

mUPD occurs when both copies of chromosome 15 are maternally inherited and the father's chromosome 15 is absent (instead of inheriting one from each parent). Therefore, neither copy of the relevant genes are active; both copies inherited form the mother are normally imprinted/switched off, and the normally active one from the father is absent.

\section{Imprinting centre defects (ID)}

As chromosome 15 has an imprinted region, the IC, the maternally inherited copy behaves differently from the paternally inherited copy. Imprinting explains why the deletions that occur in PWS always arise in the paternal copy of chromosome 15. A mutation of the IC on chromosome 15 can result in PWS. There is no loss or physical change in the relevant genes, rather their epigenetic control has been disrupted, resulting in no expression from the copy inherited from the father.

\section{Paternal copy of chromosome 15}

\begin{tabular}{|c|c|c|c|}
\hline & PWS critical region & AS critical region & \\
\hline \multicolumn{4}{|l|}{ Maternal copy of chromosome 15} \\
\hline & PWS critical region & AS critical region & \\
\hline
\end{tabular}

Figure 1. Paternally and maternally inherited chromosomes 15 denoting imprinted/ silenced regions (red) and expressed regions (green). AS=Angelman syndrome; PWS=Prader-Willi syndrome

\begin{tabular}{|c|c|c|}
\hline Subtype & Description & Results in \\
\hline \multirow[t]{2}{*}{ Deletions } & Type I BP1-BP3 & \multirow{2}{*}{$\begin{array}{l}\text { Affects the critical PWS region. The } \\
\text { chromosome is present but the PWS critical } \\
\text { region is deleted or lost; there is no active } \\
\text { copy of the genes, resulting in PWS }\end{array}$} \\
\hline & Type II BP2-BP3 & \\
\hline mUPD & $\begin{array}{l}\text { Maternal uniparental } \\
\text { disomy }\end{array}$ & $\begin{array}{l}\text { Affects the critical PWS region. The } \\
\text { chromosome is absent because of mUPD; } \\
\text { there is no active copy of the genes as the } \\
\text { maternally inherited copies have a normally } \\
\text { silenced region, resulting in PWS }\end{array}$ \\
\hline ID & $\begin{array}{l}\text { Imprinting centre } \\
\text { defects }\end{array}$ & $\begin{array}{l}\text { Affects the imprinting centre in the critical } \\
\text { PWS region. The imprinting centre is } \\
\text { present but is deleted or mutated; there } \\
\text { is no expression or function of the centre, } \\
\text { resulting in PWS }\end{array}$ \\
\hline
\end{tabular}

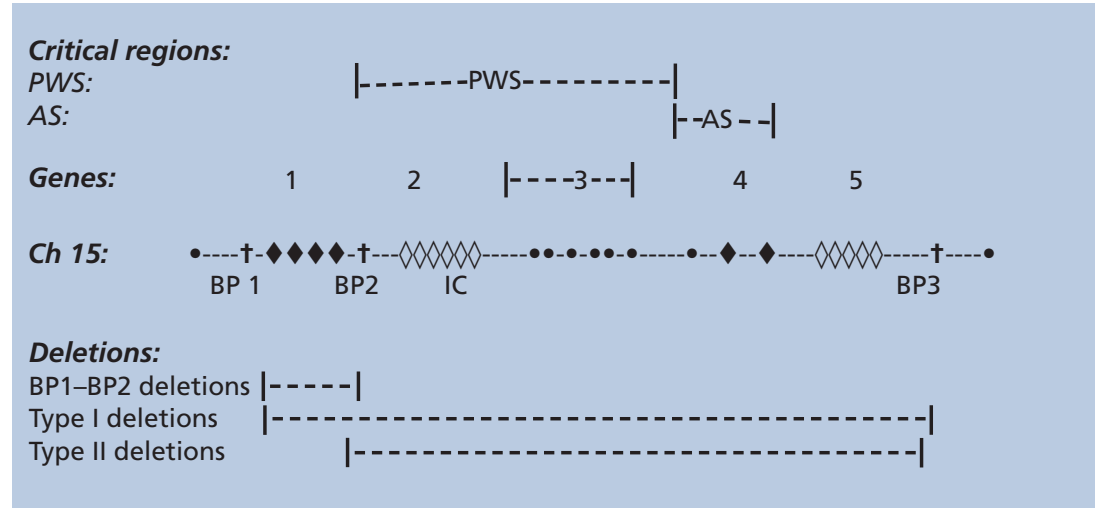

Figure 2. Genetic composition of chromosome 15 (note only key genes are denoted along the chromosome). AS=Angelman syndrome; BP1. BP2 and BP3=breakpoints; genes ( $1=$ four genes between BP1 and BP2; $2=a$ number of genes that include the imprinting centre; $3=a$ cluster of five snoRNA genes; $4=$ the UBE3A and ATP10A genes; $5=$ a cluster of gamma-aminobutyric acid (GABA) receptor genes); $\mathrm{IC}=$ imprinting centre; PWS=Prader-Willi syndrome 


\section{Other causes}

Translocations have been identified as a cause of PWS in a small number of cases, whereby there is an exchange of material between or within chromosomes, affecting chromosome 15 (Online Mendelian Inheritance in $\mathrm{Man}^{\circledR}$ $\left(\mathrm{OMIM}^{\circledR}\right)$, 2012; PWSA(UK), 2012).

\section{Regions of 15q11.2-13 and associated genes}

The 15q11.2-13 region is divided into four regions:

- A non-imprinted region between BP1 and BP2 containing four genes - a deletion involving this region has been linked with behavioural and autistic features (Butler, 2011)

- A 'PWS paternally-only expressed region' containing a number of genes, including a cluster of five snoRNA genes

- An 'AS region' containing the maternallyexpressed genes UBE3A and ATP10A, with the UBE3A being responsible for AS

- A non-imprinted region, including a cluster of three GABA receptor genes.

There are about 100 genes in the q11-13 region between $\mathrm{BP} 1$ and $\mathrm{BP} 3$, and about 10 of these genes are imprinted and paternally expressed.

Butler (2011) highlights that PWS appears not to be caused by a single locus or gene mutation, but by a deficiency of a combination of imprinted genes in the 15q11-13 region. The snoRNAs genes appear to be a key region expressing the phenotype of PWS, including the abnormal eating behaviour (Cassidy and Driscoll, 2009; McAllister et al, 2011) (Figure 2).

\section{The phenotype of PWS}

There are recognizable features associated with PWS, including neurological and developmental delay and behavioural and psychiatric difficulties (Cassidy and Driscoll, 2009; Butler, 2011; McAllister et al, 2011; McCandless and The Committee on Genetics, 2011; Sinnema et al, 2011a; OMIM ${ }^{\circledR}$, 2012; PWSA(UK), 2012). Three key features of PWS are:

- Hypotonia

- Hyperphagia

- Behavioural difficulties.

These are considered in the context of central obesity, which is a clear phenotypical feature of PWS

\section{Hypotonia}

The 'early failure to thrive' phase reflects the impact of hypotonia (low muscle tone), beginning prenatally and causing developmental delay in the prenatal and infancy periods. The hypotonia results in diminished swallowing and sucking reflexes, leading to feeding difficulties and poor weight gain.

\section{Hyperphagia}

The phase of 'childhood obesity' reflects the impact of hyperphagia (overeating). The feeding pattern of the infant is reported as changing around the age of 18 months, and by about 2 years the child will start gaining weight and will show an extreme interest in food. People with PWS appear to be lacking in the normal appetite control mechanisms and appear to have a seemingly insatiable appetite. However, the weight gain is not only caused by overeating, as people with PWS have a lower metabolic rate.

The hyperphagia is hypothalamic in origin, thought to be caused by a faulty mechanism somewhere within the hypothalamic control system. This results in a lack of sense of satiety (fullness), and therefore people with PWS continue to feel hungry even after eating more food than would be considered normal; they may eventually feel full, but will start to feel hungry again much sooner than others. Therefore, PWS should be viewed as not wanting to stop eating - a disorder of satiety rather than always wanting to start eating because of hunger (McAllister et al, 2011). Purtell and colleagues (2011) note that in healthy subjects, hunger and satiety operate in balance; when one is high, the other is low.

If food intake is not controlled, central obesity (abdominal obesity) results from this abnormal eating behaviour, combined with a low metabolic rate and decreased activity level. Theodoro et al (2006) looked at studies comparing individuals with PWS and obese subjects, and reported differences in the lean mass of trunk and limbs; people with PWS demonstrated an unusual body composition and unusual fatness patterns.

People with PWS have an extreme interest in food, as shown by common 'food seeking behaviours' such as foraging, stealing of foods, hording, eating of unappealing food items (frozen, discarded and uncooked foods) and eating of non-food items (pica). 


\section{Behavioural difficulties}

Behavioural difficulties are clearly associated with PWS, beginning in early childhood. As well as the preoccupation with food and foodseeking behaviour, other behaviours include:

- Temper outbursts

- Skin picking

- Stubbornness

- Compulsive behaviours

- Difficulties with change in routine.

\section{Phenotypic phases of PWS}

It is clear from this classic view of PWS that the key developmental stages appear to be early infancy: hypotonia from 18 months, with a change in feeding pattern; and hyperphagia from 18 months-2years, with resultant central obesity.

More recently, the phenotype of PWS has been described in terms of five phases (Table 2), with a more gradual transition between phases rather than a distinct switch (McAllister et al, 2011; Miller et al, 2011). A key difference between the traditional view and the current one is that the development of hyperphagia in the current view is around the age of 8 years, which is much older than the traditional 18 months-2years and therefore affects the care and management required for this phenotypical feature of PWS. Furthermore, in the current view it is noted that in phase $2 a$ the obesity may develop before the onset of hyperphagia in phase 3 (McAllister et al, 2011; Miller et al, 2011); this is an important issue when working with parents regarding an effective dietary regimen.

Other features have been described in PWS, including: hypogonadism, intellectual disability, a particular facial appearance, short stature as a result of growth hormone deficiency, decreased pain sensitivity, rarely vomiting and behaviour such as skin picking (Cassidy and Driscoll, 2009; Soni, 2010; Butler, 2011; McAllister et al, 2011; McCandless and The Committee on Genetics, 2011; PWSA(UK), 2012). Furthermore, the complications associated with obesity are the major causes of morbidity and mortality for people with PWS, including: cardiorespiratory insufficiency, obstructive sleep apnoea, type 2 diabetes mellitus and concerns such as choking on gorged food and rupture following binging (Blows, 2011; Brambilla et al, 2011; McAllister et al, 2011; PWSA(UK), 2012).

\section{Table 2. Five phases of PWS}

\section{Five phases \\ Description}

(with subphases in

phases 1 and 2)

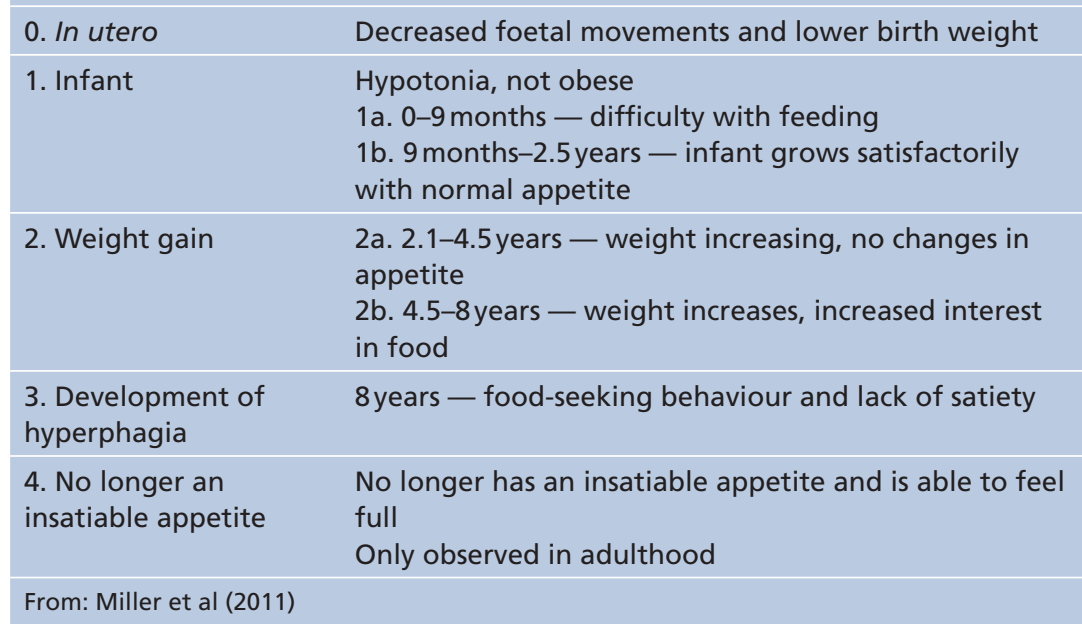

\section{Theoretical explanations for hyperphagia}

The liver, vagus nerve and hypothalamic pathway play a key role in food intake regulation; the liver relays information to the brain, and the part of the hypothalamus receiving this is dependent upon glucose and fatty acid blood levels (Zanutto and Staddon, 2007). If these levels are low, the lateral hypothalamus stimulates hunger; if these levels are high, the ventromedial hypothalamus initiates the sensation of satiety (Williams et al, 2000). Blows (2011) discusses the role of various parts of the brain and the role of leptin (a hormone released by adipose) in food regulation.

The exact aetiology of the transition from poor feeding and failure to thrive seen in the early phases to the obesity and hyperphagia of the later phases has yet to be established, but it is thought that the hyperphagia and endocrine difficulties are caused by generalized hypothalamic dysfunction (McAllister, 2011; Miller et al, 2011; PWSA(UK), 2012).

Research into the role of hormones and proteins in PWS aims to ascertain the precise underlying dysfunction to determine potential treatments; however, no clear understanding exists and reports are often contradictory (Purtell et al, 2011).

Recent research topics include:

- Insulin sensitivity - this is higher in people with PWS; however, the role of insulin in PWS remains obscure with a suggestion of a differing role in 
PWS (Haqq et al, 2011), although no definite evidence is available (Purtell et al, 2011)

- Ghrelin - this is an orexogenic hormone produced in the stomach and pancreas that increases appetite; higher levels have been reported in people with PWS (Miller et al, 2011; Purtell et al, 2011; PWSA(UK), 2012) and $Y i$ et al (2011) reported altered levels, but McAllister and colleagues (2011) suggest that the role in PWS has yet to be fully established

- Peptide YY (PYY) - this is a pancreatic polypeptide with anorexigenic actions (appetite suppressant); low levels of this hormone are reported in people with PWS (PWSA(UK), 2012), although its role in PWS has yet to be established (Purtell et al, 2011)

- Glycogen-like peptide-1 (GLP-1) - this has several functions, including increasing insulin secretion and satiety; however, its role in PWS has yet to be established (Purtell et al, 2011)

- Fat distribution in people with PWS differences in fat distribution can influence hormone levels; however, in PWS there is currently no clear understanding (Haqq et al, 2011; Purtell et al, 2011)

- Genetics - no direct link has been established to genes located at 15q11.2-13; it is therefore suggested that the consequence of genetics on the hypothalamic feeding pathway and the role of the prenatal environment remain key research areas (McAllister et al, 2011).

\section{Care and management of PWS} A key priority is early identification and a differential diagnosis, as there are several disorders that can resemble PWS. Once the diagnosis has been established, appropriate care and management can be put in place. This consists of proactive (anticipatory) and reactive care strategies, management and guidance in terms of responding to the various phenotypical features of PWS at specific age-dependent stages. As people with PWS have a shorter life expectancy, the literature depicting the care of people with PWS in later adulthood is limited. Thus people reaching this lifetime stage are often cared for in a more reactive manner in terms of the emergence of any new phenotypical features.

\section{Proactive/anticipatory strategies}

Proactive strategies can be put in place as the early phenotypical features of PWS, such as hypotonia and hyperphagia, are well documented. Proactive strategies include:

- Assisted feeding to overcome feeding difficulties, such as with feeding tubes (nasogastric)

- Establishing a regular feeding pattern to ensure adequate nutrition as infants with PWS rarely wake to feed or are undemanding. Proactive strategies for hyperphagia include:

- A dietary regimen, such as regular meal times with a calorie-balanced diet but with limited portion sizes

- The secure containment of food, which may necessitate locking the refrigerator and food cupboards and notifying others to do this, including the school and other relevant carers

- Responding to the features as they arise, such as the stealing and foraging of foods and pica, as the impact of hyperphagia may dominate the lives of people with PWS as well as their families and carers

- As a result of the erratic nature of the eating patterns in people with PWS, McCandless and The Committee on Genetics (2011) note that parents should be informed that food offered as a reward or the withholding of food as a punishment is counterproductive and should be avoided.

There is no effective medication available for hyperphagia and obesity (Cassidy and Driscoll, 2009; Butler, 2011; McAllister et al, 2011; Purtell et al, 2011; PWSA(UK), 2012). However, Cassidy and Driscoll (2009) and the PWSA(UK) (2012) report that dramatic benefits can be achieved for people with PWS by appropriate care and management, in terms of the phenotype, health and self-image. The severity of the obesity can be managed by early diagnosis, diet restrictions, controlling access to food and tailored exercise programmes (Sinnema et al, 2011b). However, weight control by strictly restricting calorific intake may lead to physiological and psychological stress (Dykens et al, 2011; Grolla et al, 2011). Grolla and colleagues (2011) have reported successful weight loss and weight control by exercise and physical therapies without strict dietary restrictions.

The improvement and benefits of growth hormone therapy has been demonstrated, although undesired side effects and controversies over their use have been reported (Cassidy and Driscoll, 2009; Butler, 2011; McCandless and 
The Committee on Genetics, 2011).

Advice and guidance is available from associations such as the Prader-Willi Syndrome Association UK (2012) and the Prader-Willi Syndrome Association USA (2012).

\section{Psychological implications}

The features of PWS have not only practical implications but psychological ones as well for the people with PWS, their family members and carers; each phenotypical feature (symptom) merits consideration in its own right. PWS can affect self-image and self-esteem, and further lead to psychiatric difficulties such as depression. The resultant impaired cognitive functioning in PWS further complicates the psychological implications, as people with PWS experience impairment of cognitive processes that hinder their understanding and managing of their own phenotypical features and associated behaviours.

The hyperphagia and constant foraging for food can overshadow developmental behaviours; it is therefore important to consider the child/adolescent developmental stages and life experiences, rather than fixating on this feature of PWS. The phenotypic features of the condition and the restrictions that these place on the life experiences of the person with PWS and other family members should therefore be of central concern to all involved in that person's care and management.

A multidisciplinary team approach is essential in addressing the varying needs of the person with PWS and family, and should include physical, speech and occupational therapists, a dietician, education, counselling and psychiatric assessment/treatment (Goldstone et al, 2008). Care strategies are required throughout the lifetime of the person with PWS, as the phenotypic features are lifelong and therefore necessitate specific age-dependent interventions.

Blows WT (2011) The physiology of food intake regulation and eating disorders. Gastrointest Nurs 9(6): 40-5

Brambilla P, Crinò A, Bedogni G et al (2011) Metabolic syndrome in children with Prader-Willi syndrome: the effect of obesity. Nutr Metab Cardiovas Dis 21(4): 269-76

Butler MG (2011) Prader-Willi syndrome: obesity due to genomic imprinting. Curr Genomics 12(3): 204-15

Cassidy SB, Driscoll DJ (2009) Prader-Willi syndrome. Eur J Hum Genet 17(1): 3-13

DuBose AJ, Smith EY, Yang TP, Johnstone KA, Resnick JL (2011) A new deletion refines the boundaries of the murine Prader-Willi syndrome imprinting center. Hum Mol
Genet 20(17): 3461-6

Dykens EM, Lee E, Roof E (2011) Prader-Willi syndrome and autism spectrum disorders: an evolving story. J Neurodev Disord 3(3): 225-37

Goldstone AP (2004) Prader-Willi syndrome: advances in genetics, pathophysiology and treatment. Trends Endocrinol Metab 15(1): 12-20

Goldstone AP, Holland AJ, Hauffa BP, Hokken-Koelega AC, Tauber M (2008) Recommendations for the diagnosis and management of Prader-Willi syndrome. J Clin Endocrinol Metab 93(11): 4138-97

Grolla E, Andrighetto G, Parmigiani P et al (2011) Specific treatment of Prader-Willi syndrome through cyclical rehabilitation programmes. Disabil Rehabil 33:1837-47

Haqq AM, Muehlbauer J, Newgard CB, Grambow S, Freemark M (2011) The metabolic phenotype of PraderWilli syndrome in childhood: heightened insulin sensitivity relative to body mass index. J Clin Endocrinol Metab 96(1): E225-32

McAllister CJ, Whittington JE, Holland AJ (2011) Development of the eating behaviour in Prader-Willi syndrome: advances in our understanding. Int J Obes 35(2): 188-97

McCandless SE, The Committee on Genetics (2011) Clinical report: health supervision for children with Prader-Willi syndrome. Pediatrics 127(1): 195-205

Miller JL, Lynn CH, Driscoll DC et al (2011) Nutritional phases in Prader-Willi syndrome. Am J Med Genet A 155: 1040-9

Online Mendelian Inheritance in $\operatorname{Man}^{\circledast}\left(\mathrm{OMIM}^{\circledR}\right)$ (2012) Prader-Willi Syndrome. http://omim.org/entry/176270

Prader A, Labhart A, Willi H (1956) Ein sydnrom von adipositas, kleinwuchs, kryptorchismus und oligophrenie nach myatonieartigem zustand im neugeborenenalter. Schweizerische Medizinishce Wochenschrift 6(3): 1260-1

Prader-Willi Syndrome Association UK (PWSA(UK)) (2012) Prader-Willi Syndrome. http://www.pwsa.co.uk

Prader-Willi Syndrome Association USA (PWSA(USA)) (2012) Prader-Willi Syndrome. http://www.pwsausa.org/

Purtell L, Sze L, Loughnan G et al (2011) In adults with Prader-Willi syndrome, elevated ghrelin levels are more consistent with hyperphagia than high PYY and GLP-1 levels. Neuropeptides 45(4): 301-7

Sinnema M, Einfield SL, Schrander-Stumple CTRM, Maaskant MA, Boer H, Curfs LMG (2011a) Behavioral phenotype in adults with Prader-Willi syndrome. Res Dev Disabil 32(2): 604-12

Sinnema M, Maaskant MA, van Schrojenstein Lantman-de Valk HMJ et al (2011b) Physical health problems in adults with Prader-Willi syndrome. Am J Med Genet A 155(9): 2112-24.

Soni S (2010) Prader-Willi Syndrome. Society for the Study of Behavioural Phenotypes. www.ssbp.org.uk

Theodoro MF, Talebizadeh Z, Butler MG (2006) Body composition and fatness patterns in Prader-Willi syndrome: comparison with simple obesity. Obesity 14(10): 1685-90

Williams G, Harrold JA, Cutler DJ (2000) The hypothalamus and the regulation of energy homeostasis: lifting the lid on a black box. Proc Nutr Soc 59(3): 385-96

Yi C-X, Heppner K, Tschöp MH (2011) Ghrelin in eating disorders. Mol Cell Endocrinol 340(1): 29-34

Zanutto BS, Staddon JER (2007) Bang-bang control of feeding: role of hypothalamic and satiety signals. PLOS Comput Bio/ 3(5): e97. doi:10.1371/journal.pcbi.0030097 